\title{
Solving Robin problems in multiply connected regions via an integral equation with the generalized Neumann kernel
}

\author{
Shwan HH Al-Shatri ${ }^{1}$, Ali HM Murid ${ }^{1,2^{*}}$, Munira Ismail ${ }^{1}$ and Mukhiddin I Muminov ${ }^{1}$
}

\author{
"Correspondence: \\ alihassan@utm.my \\ 'Department of Mathematical \\ Sciences, Faculty of Science, \\ Universiti Teknologi Malaysia, Johor \\ Bahru, Johor, 81310, Malaysia \\ ${ }^{2}$ UTM Centre for Industrial and \\ Applied Mathematics (UTM-CIAM), \\ Ibnu Sina Institute for Scientific and \\ Industrial Research, University \\ Teknologi Malaysia, Johor Bahru, \\ Johor, 81310, Malaysia
}

\begin{abstract}
This paper presents a boundary integral equation method for finding the solution of Robin problems in bounded and unbounded multiply connected regions. The Robin problems are formulated as Riemann-Hilbert problems which lead to systems of integral equations and the related differential equations are also constructed that give rise to unique solutions, which are shown. Numerical results on several test regions are presented to illustrate that the approximate solution when using this method for the Robin problems when the boundaries are sufficiently smooth are accurate.
\end{abstract}

Keywords: Robin problem; Riemann-Hilbert problem; integral equation; generalized Neumann kernel; multiply connected region

\section{Introduction}

A boundary value problem is a problem that involves finding the solution of a differential equation or system of differential equation which meets certain specified requirements or boundary conditions at the end points or along a boundary, usually connected with the physical condition for certain values of the independent variable. This paper considers Laplace's equation $\Delta u=0$ in both bounded and unbounded multiply connected regions $\Omega$ with a linear combination of Dirichlet and Neumann boundary conditions on the boundary $\Gamma=\partial \Omega$, generally known as a mixed boundary value problem and commonly called the Robin problem.

Applications of mixed boundary value problems exist in large numbers in classical mathematical physics, physical geodesy, electro-magnetics, analysis of measurement [1,2], and specific boundary problems such as the Dirichlet problem and the Neumann problem [3]. The applications of the mixed boundary value problem in potential theory can be found in [4].

It has been shown that the problem of conformal mapping, the Dirichlet problem, the Neumann problem, and the mixed Dirichlet-Neumann problem can all be treated as Riemann Hilbert (briefly RH) problems as discussed in [5-7]. The interplay of RH problems and the boundary Fredholm integral equation with the generalized Neumann kernel has been investigated in [8] for simply connected regions with smooth and piecewise smooth boundaries and in [9] for bounded and unbounded multiply connected regions.

(c) 2016 Al-Shatri et al. This article is distributed under the terms of the Creative Commons Attribution 4.0 International License (http://creativecommons.org/licenses/by/4.0/), which permits unrestricted use, distribution, and reproduction in any medium, provided you give appropriate credit to the original author(s) and the source, provide a link to the Creative Commons license, and indicate if changes were made. 
Earlier, the well-known integral equations for RH problem have been employed for solving the Dirichlet problem and the Neumann problem [5] and the mixed DirichletNeumann problem [6]. They are uniquely solvable Fredholm integral equations of the second kind. However, the problems in [5] and [6] are not Robin problems, since the Dirichlet condition and Neumann condition are given separately.

This paper solves the Robin problem by reducing it to a RH problem and hence providing a related system of boundary integral equations. Additional conditions are given to obtain a unique solution to the Robin problem.

This paper is organized as follows: After the presentation of some notations and auxiliary material in Section 2, we present in Section 3, the reduction of the Robin problems in bounded and unbounded multiply connected regions to the $\mathrm{RH}$ problem. We then show how to construct the integral equations and differential equations related to the Robin problems. Conditions for obtaining unique solution for the Robin problems are also given. In Section 4, we show how to treat the integral equations and differential equations numerically. In Section 5, we give some numerical examples for several test regions. In Section 6 we give a short conclusion.

\section{Notations and auxiliary material}

Consider a multiply connected region $\Omega$ in the extended complex plane $\overline{\mathbb{C}}$ of the following two types [5]:

(a) A bounded region $\Omega$ of connectivity $m+1 \geq 1$ with boundary $\Gamma=\bigcup_{j=0}^{m} \Gamma_{j}$ consisting of $m+1$ smooth closed Jordan curves $\Gamma_{j}, j=1,2, \ldots, m$. The curve $\Gamma_{0}$ contains the other curves $\Gamma_{1}, \ldots, \Gamma_{m}$. The complement $\Omega^{-}:=\overline{\mathbb{C}} \backslash \bar{\Omega}$ consists of $m$ bounded simply connected components $\Omega_{j}$ interior to $\Gamma_{j}, j=1,2, \ldots, m$, and an unbounded simply connected component $\Omega_{0}$ exterior to $\Gamma_{0}$ (see Figure 1). We assume that $0 \in \Omega$.

(b) An unbounded region $\Omega$ of connectivity $m \geq 1$ with boundary $\Gamma=\bigcup_{j=1}^{m} \Gamma_{j}$ consisting of $m$ smooth closed Jordan curves $\Gamma_{j}, j=1,2, \ldots, m$. The complement $\Omega^{-}:=\overline{\mathbb{C}} \backslash \bar{\Omega}$ consists of $m$ bounded simply connected components $\Omega_{j}$ interior to $\Gamma_{j}, j=1,2, \ldots, m$ (see Figure 2). We assume that $0 \notin \Omega$.

The orientation of the boundary $\Gamma=\partial \Omega$ is such that $\Omega$ is always on the left of $\Gamma$. Thus, the curves $\Gamma_{1}, \ldots, \Gamma_{m}$ always have clockwise orientations. For bounded $\Omega$, the curve $\Gamma_{0}$ has a counterclockwise orientation. The curves $\Gamma_{j}$ are parameterized by $2 \pi$-periodic twice

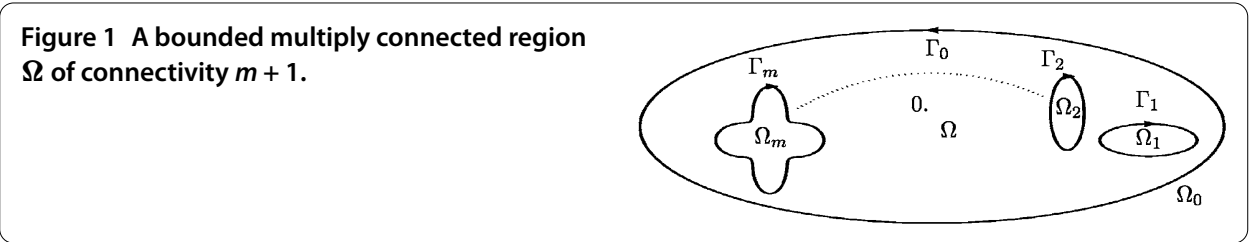

Figure 2 An unbounded multiply connected region $\Omega$ of connectivity $m$.

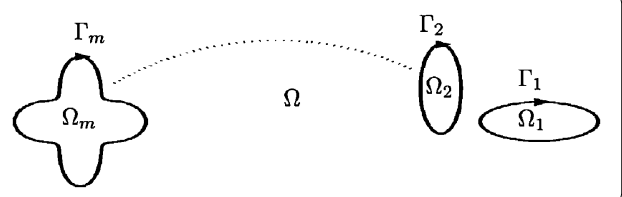


continuously differentiable complex functions $\eta_{j}(t)$ with non-vanishing first derivatives,

$$
\dot{\eta}_{j}(t)=\frac{d \eta_{j}(t)}{d t} \neq 0, \quad t \in J_{j}:=[0,2 \pi],
$$

$j=0$ (for bounded $\Omega), 1,2, \ldots, m$. We represent the disjoint union of the intervals $J_{j}$ by $J$ as a total parameter domain. Automatically, the whole boundary $\Gamma$ will be parameterized by a complex function $\eta$ defined on $J$ by

$$
\eta(t):= \begin{cases}\eta_{0}(t), & t \in J_{0}(\text { for bounded } \Omega), \\ \eta_{1}(t), & t \in J_{1}, \\ \vdots & \\ \eta_{m}(t), & t \in J_{m} .\end{cases}
$$

Assume that $H$ be the space of all real Hölder continuous functions on the boundary $\Gamma$. Since $\eta$ is smooth, a function $\phi \in H$ can be interpreted via $\hat{\phi}(t):=\phi(\eta(t)), t \in J$, as a real Hölder continuous $2 \pi$-periodic functions $\hat{\phi}(t)$ of the parameter $t \in J$, i.e.,

$$
\hat{\phi}(t):= \begin{cases}\hat{\phi}_{0}(t), & t \in J_{0}(\text { for bounded } \Omega), \\ \hat{\phi}_{1}(t), & t \in J_{1}, \\ \vdots & \\ \hat{\phi}_{m}(t), & t \in J_{m},\end{cases}
$$

with real Hölder continuous $2 \pi$-periodic functions $\hat{\phi}_{j}$ defined on $J_{j}$.

From now on, for a complex-valued or a real-valued function $\psi \in H$ defined on the boundary $\Gamma$ and for $t \in J$, we will not distinguish between $\psi(\eta(t))$ and $\psi(t)$. For $t \in J_{k}$, the values $\psi(t)$ will be denoted by $\psi_{k}(t)$.

For given functions $\alpha \in H, \beta \in H, l \in H$, a Robin problem is a boundary value problem for determining a harmonic function $u(x, y)$ harmonic in $\Omega$ and continuous on $\Omega \cup \Gamma$ and satisfying the Robin boundary condition [10]

$$
\alpha(t) u(\eta(t))+\beta(t) \frac{\partial u(\eta(t))}{\partial \mathbf{n}}=l(t), \quad \alpha(t) \neq 0, \beta(t) \neq 0, \eta(t) \in \Gamma,
$$

where $\mathbf{n}$ is an exterior normal to $\Gamma$. In particular, if $t \in J_{k}$, then (1) becomes

$$
\alpha_{k}(t) u\left(\eta_{k}(t)\right)+\beta_{k}(t) \frac{\partial u\left(\eta_{k}(t)\right)}{\partial \mathbf{n}}=l_{k}(t), \quad \alpha_{k}(t) \neq 0, \beta_{k}(t) \neq 0, \eta_{k}(t) \in \Gamma, t \in J_{k} .
$$

For unbounded $\Omega$, the function $u$ is also required to satisfy $u(z) \rightarrow C$ as $|z| \rightarrow \infty$ with a constant $C$. If $\frac{\alpha_{k}(t)}{\beta_{k}(t)}>0, t \in J_{k}$ (briefly $\frac{\alpha(t)}{\beta(t)}>0$ ), then the Robin problem is uniquely solvable (see e.g. [11], p.163, [12], p.103, [13], pp.314-315, and [14], p.141). For some integral equations related to the Robin problem, see [13], p.314, and [15]. Without loss of generality, we assume $C=0$.

In this paper, we shall relate the Robin problem with the RH problem. The RH problem consists of finding a function $g$ analytic in $\Omega$, continuous in its closure $\bar{\Omega}$ and having boundary values

$$
\operatorname{Re}[A g]=\gamma,
$$


where $\gamma \in H$ and $A(t)$ is a complex continuously differentiable $2 \pi$-periodic function with $A \neq 0$ for all $t$ such that

$$
A(t):= \begin{cases}A_{0}(t), & t \in J_{0}(\text { for bounded } \Omega), \\ A_{1}(t), & t \in J_{1}, \\ \vdots & \\ A_{m}(t), & t \in J_{m} .\end{cases}
$$

For unbounded $\Omega$, the function $g$ is also required to satisfy $g(\infty)=0$.

The $\mathrm{RH}$ problem can be solved using a boundary integral equation with the generalized Neumann kernel. Define the real kernels $M$ and $N$ as real and imaginary parts [8, 9]:

$$
\begin{array}{ll}
M(\tau, t)=\frac{1}{\pi} \operatorname{Re}\left[\frac{A(\tau)}{A(t)} \frac{\dot{\eta}(t)}{\eta(t)-\eta(\tau)}\right], & \tau \neq t, \\
N(\tau, t)=\frac{1}{\pi} \operatorname{Im}\left[\frac{A(\tau)}{A(t)} \frac{\dot{\eta}(t)}{\eta(t)-\eta(\tau)}\right], & \tau \neq t .
\end{array}
$$

The kernel $N(\tau, t)$ is called the generalized Neumann kernel formed with $A$ and $\eta$. When $A=1$, it reduces to the classical Neumann kernel,

$$
N(\tau, t)=\frac{1}{\pi} \operatorname{Im}\left[\frac{\dot{\eta}(t)}{\eta(t)-\eta(\tau)}\right], \quad \tau \neq t .
$$

The generalized Neumann kernel (4) is continuous at $t=\tau$ with

$$
N(t, t)=\frac{1}{\pi} \operatorname{Im}\left[\frac{1}{2}\left(\frac{\ddot{\eta}(t)}{\dot{\eta}(t)}\right)-\frac{\dot{A}(t)}{A(t)}\right] .
$$

The kernel $M(\tau, t)$ has the representation

$$
M(\tau, t)=-\frac{1}{2 \pi} \cot \frac{\tau-t}{2}+M_{1}(\tau, t), \quad t \neq \tau,
$$

with the continuous kernel $M_{1}$, which takes on the diagonal the values

$$
M_{1}(t, t)=\frac{1}{\pi} \operatorname{Re}\left[\frac{1}{2}\left(\frac{\ddot{\eta}(t)}{\dot{\eta}(t)}\right)-\frac{\dot{A}(t)}{A(t)}\right] .
$$

For details, see $[8,9]$.

Let $\mathbf{N}$ and $\mathbf{M}_{1}$ be the Fredholm integral operators associated with the continuous kernels $N$ and $M_{1}$, i.e.,

$$
\begin{aligned}
& (\mathbf{N} \mu)(\tau)=\int_{J} N(\tau, t) \mu(t) d t, \quad \tau \in J, \\
& \left(\mathbf{M}_{1} \mu\right)(\tau)=\int_{J} M_{1}(\tau, t) \mu(t) d t, \quad \tau \in J .
\end{aligned}
$$

Let $\mathbf{M}$ and $\mathbf{K}$ be the singular integral operators

$$
(\mathbf{M} \mu)(\tau)=\int_{J} M(\tau, t) \mu(t) d t, \quad \tau \in J
$$




$$
(\mathbf{K} \mu)(\tau)=\frac{1}{2 \pi} \int_{J_{k}} \cot \left(\frac{\tau-t}{2}\right) \mu(t) d t, \quad \tau \in J_{k}, k=0,1, \ldots, m
$$

The integrals (6) and (7) are principal value integrals. The operator $\mathbf{K}$ is known as the conjugation operator. It is also known as the Hilbert transform [8]. It follows from equation (5) that

$$
\mathbf{M}=\mathbf{M}_{1}-\mathbf{K} .
$$

Theorem $1([9,16])$ If $g$ is a solution of the RH problem equation (3) with boundary values

$$
A g=\gamma+\mathrm{i} \mu,
$$

then the imaginary part $\mu$ in (8) satisfies the integral equation

$$
\mu-\mathbf{N} \mu=-\mathbf{M} \gamma
$$

and the real part $\gamma$ in (8) satisfies the integral equation

$$
\gamma-\mathbf{N} \gamma=\mathbf{M} \mu
$$

The solvability of boundary integral equations with the generalized Neumann kernel is determined by the index (winding number in other terminology) of the function $A(t)$ [9].

Theorem 2 ([17] Cauchy integral formula) Let $f$ be a function that is analytic everywhere in $\Omega$ and on a simple closed contour $\Gamma$. Then the Cauchy integral formula is given by

$$
\begin{aligned}
& \frac{1}{2 \pi \mathrm{i}} \int_{\Gamma} \frac{f(\eta)}{\eta-z} d \eta=\left\{\begin{array}{ll}
f(z), & z \in \Omega, \\
0, & z \notin \Omega
\end{array} \quad[\text { Bounded } \Omega],\right. \\
& \frac{1}{2 \pi \mathrm{i}} \int_{\Gamma} \frac{f(\eta)}{\eta-z} d \eta=\left\{\begin{array}{ll}
f(z)-f(\infty), & z \in \Omega, \\
-f(\infty), & z \notin \Omega
\end{array} \quad \text { [Unbounded } \Omega\right] .
\end{aligned}
$$

\section{Reduction Robin problems in bounded and unbounded multiply connected regions to Riemann-Hilbert problem}

We consider the Robin problem (1) either bounded or unbounded. The unit exterior normal vector is given by $\mathbf{n}(\eta(t))=e^{-\mathrm{i} \frac{\pi}{2}} \mathbf{T}(\eta(t))=-\mathrm{i} \frac{\dot{\eta}(t)}{|\dot{\eta}(t)|}=e^{\mathrm{i} \theta(\eta(t))}=\cos \theta(\eta(t))+\mathrm{i} \sin \theta(\eta(t))$.

Then

$$
\begin{aligned}
\frac{\partial u(\eta(t))}{\partial \mathbf{n}} & =\nabla u \cdot \mathbf{n}=\left(u_{x} \overrightarrow{\mathbf{i}}+u_{y} \overrightarrow{\mathrm{j}}\right) \cdot(\cos \theta(\eta(t)) \overrightarrow{\mathrm{i}}+\mathrm{i} \sin \theta(\eta(t)) \overrightarrow{\mathrm{j}}) \\
& =\cos \theta(\eta(t)) \frac{\partial u(\eta(t))}{\partial x}+\sin \theta(\eta(t)) \frac{\partial u(\eta(t))}{\partial y} \\
& =\operatorname{Re}\left[e^{\mathrm{i} \theta(\eta(t))}\left(\frac{\partial u(\eta(t))}{\partial x}-\mathrm{i} \frac{\partial u(\eta(t))}{\partial y}\right)\right] .
\end{aligned}
$$

Assume $u(z)=\operatorname{Re}[f(z)]$, where $f(z)=u+\mathrm{i} v$, is analytic in $\Omega$. For bounded $\Omega$, we further assume $\operatorname{Im}[f(0)]=0$, for unbounded $\Omega$, we assume $f(\infty)=0$. Then by the Cauchy-Riemann 
equations we have $f^{\prime}(z)=u_{x}-\mathrm{i} u_{y}$. Thus,

$$
\frac{\partial u(\eta(t))}{\partial \mathbf{n}}=\operatorname{Re}\left[\frac{-\mathrm{i} \dot{\eta}(t) f^{\prime}(\eta(t))}{|\dot{\eta}(t)|}\right] .
$$

Substituting this result into the Robin condition (1), we get

$$
\alpha(t) \operatorname{Re}[f(\eta(t))]-\beta(t) \operatorname{Re}\left[\frac{\mathrm{i} \dot{\eta}(t) f^{\prime}(\eta(t))}{|\dot{\eta}(t)|}\right]=l(t) .
$$

It is sometimes assumed that $\eta_{j}(t)$ are the arc length parameterization of the boundary $\Gamma_{j}$, which implies $\left|\eta_{j}(t)\right|=1$. These assumptions, although convenient for theoretical work, but in numerical aspect, it introduces an additional sources of error [18]. If $\Omega$ is a unit disk (11) yields the same result as in Petrila [10]. Multiplying both sides of (11) by $|\dot{\eta}(t)|$ we get

$$
\operatorname{Re}\left[\alpha(t)|\dot{\eta}(t)| f(\eta(t))-\mathrm{i} \beta(t) \dot{\eta}(t) f^{\prime}(\eta(t))\right]=l(t)|\dot{\eta}(t)|,
$$

or, equivalently,

$$
\operatorname{Re}\left[-\mathrm{i} \beta(t)\left\{\frac{d}{d t}(f(\eta(t)))+\mathrm{i} e(t) f(\eta(t))\right\}\right]=l(t)|\dot{\eta}(t)|,
$$

where

$$
e(t)=\frac{\alpha(t)}{\beta(t)}|\dot{\eta}(t)|, \quad t \in J .
$$

Hence

$$
-\mathrm{i} \beta(t)\left[\frac{d}{d t}(f(\eta(t)))+\mathrm{i} e(t) f(\eta(t))\right]=l(t)|\dot{\eta}(t)|+\mathrm{i} \mu(t), \quad t \in J,
$$

where

$$
\mu(t)= \begin{cases}\hat{\mu}_{0}(t), \quad t \in J_{0}(\text { for bounded } \Omega), \\ \hat{\mu}_{1}(t), \quad t \in J_{1}, \\ \vdots & \\ \hat{\mu}_{m}(t), & t \in J_{m},\end{cases}
$$

is an unknown function. By means of integrating factor, we obtain

$$
\begin{aligned}
& -\mathrm{i} \beta(t) A(t)\left[\frac{d}{d t}(f(\eta(t)))+\mathrm{i} e(t) f(\eta(t))\right]=l(t)|\dot{\eta}(t)| e^{\mathrm{i} \zeta(t)}+\mathrm{i} \mu(t) e^{\mathrm{i} \zeta(t)}, \quad t \in J, \\
& A(t)=e^{\mathrm{i} \int_{0}^{t} e(\tau) d \tau}=e^{\mathrm{i} \zeta(t)} \\
& \zeta(t)=\int_{0}^{t} e(\tau) d \tau .
\end{aligned}
$$

Then equation (12) becomes

$$
\frac{d}{d t}[A(t)(-\mathrm{i})(f(\eta(t)))]=\frac{l(t)|\dot{\eta}(t)| e^{\mathrm{i} \zeta(t)}+\mathrm{i} \mu(t) e^{\mathrm{i} \zeta(t)}}{\beta(t)} .
$$


Letting $g=-\mathrm{i} f$, which is analytic on $\Omega$, we obtain

$$
\frac{d}{d t}[A(t) g(\eta(t))]=\frac{l(t)|\dot{\eta}(t)| e^{\mathrm{i} \zeta(t)}+\mathrm{i} \mu(t) e^{\mathrm{i} \zeta(t)}}{\beta(t)} .
$$

Hence, it follows from (13) that

$$
\begin{aligned}
A(t) g(\eta(t))= & \int_{0}^{t} \frac{l(\tau)|\dot{\eta}(\tau)| \cos \zeta(\tau)}{\beta(\tau)} d \tau+\mathrm{i} \int_{0}^{t} \frac{l(\tau)|\dot{\eta}(\tau)| \sin \zeta(\tau)}{\beta(\tau)} d \tau \\
& +\mathrm{i} \int_{0}^{t} \frac{\mu(\tau) \cos \zeta(\tau)}{\beta(\tau)} d \tau-\int_{0}^{t} \frac{\mu(\tau) \sin \zeta(\tau)}{\beta(\tau)} d \tau+c_{1}+\mathrm{i} c_{2} \\
= & \gamma_{1}(t)+\mathrm{i} \gamma_{2}(t)+\mathrm{i} \mu_{1}(t)-\mu_{2}(t)+c_{1}+\mathrm{i} c_{2} \\
= & \left(\gamma_{1}(t)-\mu_{2}(t)+c_{1}\right)+\mathrm{i}\left(\gamma_{2}(t)+\mu_{1}(t)+c_{2}\right), \quad t \in J,
\end{aligned}
$$

where $c_{1}, c_{2}$, are unknown piecewise real constants, and

$$
\begin{aligned}
& \gamma_{1}(t):=\int_{0}^{t} \frac{l(\tau)|\dot{\eta}(\tau)| \cos \zeta(\tau)}{\beta(\tau)} d \tau, \quad t \in J, \\
& \gamma_{2}(t):=\int_{0}^{t} \frac{l(\tau)|\dot{\eta}(\tau)| \sin \zeta(\tau)}{\beta(\tau)} d \tau, \quad t \in J
\end{aligned}
$$

are known functions, and

$$
\begin{aligned}
& \mu_{1}(t):=\int_{0}^{t} \frac{\mu(\tau) \cos \zeta(\tau)}{\beta(\tau)} d \tau, \quad t \in J, \\
& \mu_{2}(t):=\int_{0}^{t} \frac{\mu(\tau) \sin \zeta(\tau)}{\beta(\tau)} d \tau, \quad t \in J
\end{aligned}
$$

are unknown functions. Define

$$
\begin{gathered}
c_{1}= \begin{cases}c_{01}, & \left.t \in J_{0}=[0,2 \pi] \text { (for bounded } \Omega\right), \\
c_{11}, & t \in J_{1}=[0,2 \pi], \\
\vdots & \\
c_{m 1}, & t \in J_{m}=[0,2 \pi],\end{cases} \\
c_{2}= \begin{cases}c_{02}, & \left.t \in J_{0}=[0,2 \pi] \text { (for bounded } \Omega\right), \\
c_{12}, & t \in J_{1}=[0,2 \pi], \\
\vdots & \\
c_{m 2}, & t \in J_{m}=[0,2 \pi],\end{cases} \\
\gamma_{1}(t)= \begin{cases}\gamma_{01}(t), & t \in J_{0}=[0,2 \pi](\text { for bounded } \Omega), \\
\gamma_{11}(t), & t \in J_{1}=[0,2 \pi], \\
\vdots & \\
\gamma_{m 1}(t), & t \in J_{m}=[0,2 \pi],\end{cases}
\end{gathered}
$$




$$
\begin{aligned}
& \gamma_{2}(t)= \begin{cases}\gamma_{02}(t), & t \in J_{0}=[0,2 \pi](\text { for bounded } \Omega), \\
\gamma_{12}(t), & t \in J_{1}=[0,2 \pi], \\
\vdots & \\
\gamma_{m 2}(t), & t \in J_{m}=[0,2 \pi],\end{cases} \\
& \mu_{1}(t)= \begin{cases}\mu_{01}(t), & t \in J_{0}=[0,2 \pi](\text { for bounded } \Omega), \\
\mu_{11}(t), & t \in J_{1}=[0,2 \pi], \\
\vdots & \\
\mu_{m 1}(t), & t \in J_{m}=[0,2 \pi],\end{cases} \\
& \mu_{2}(t)= \begin{cases}\mu_{02}(t), & t \in J_{0}=[0,2 \pi](\text { for bounded } \Omega), \\
\mu_{12}(t), & t \in J_{1}=[0,2 \pi], \\
\vdots & \\
\mu_{m 2}(t), & t \in J_{m}=[0,2 \pi] .\end{cases}
\end{aligned}
$$

Then we can write (14) briefly as

$$
A(t) g(t)=\gamma(t)+\mathrm{i} \mu(t), \quad t \in J
$$

where $g(t)=g(\eta(t))$,

$$
\begin{aligned}
& \gamma(t)=\gamma_{1}(t)-\mu_{2}(t)+c_{1}, \\
& \mu(t)=\gamma_{2}(t)+\mu_{1}(t)+c_{2} .
\end{aligned}
$$

The real part of (19) yields the RH problem. The function $A(t)=e^{\mathrm{i} \zeta(t)}$ is in general not periodic. To apply the result of Theorem $1, A(t)$ must be periodic. The function $A(t)$ is periodic if we assume $\zeta(2 \pi)-\zeta(0)=2 \pi$. Thus, Theorem 1 implies that (19) can be reformulated as

$$
\begin{aligned}
& (\mathbf{I}-\mathbf{N})\left(\gamma_{2}(t)+\mu_{1}(t)+c_{2}\right)=-\mathbf{M}\left(\gamma_{1}(t)-\mu_{2}(t)+c_{1}\right), \quad t \in J, \\
& (\mathbf{I}-\mathbf{N})\left(\gamma_{1}(t)-\mu_{2}(t)+c_{1}\right)=\mathbf{M}\left(\gamma_{2}(t)+\mu_{1}(t)+c_{2}\right), \quad t \in J .
\end{aligned}
$$

We next show that the above system of integral equations are linearly independent.

Theorem 3 Let $\frac{\alpha(t)}{\beta(t)}>0$. Then the following system of integral equations are linearly independent:

$$
\begin{aligned}
& (\mathbf{I}-\mathbf{N}) x=-\mathbf{M} y, \\
& (\mathbf{I}-\mathbf{N}) y=\mathbf{M} x
\end{aligned}
$$

for $x, y \in H$.

Proof We define the functions $\mathrm{S}$ and $\mathrm{T}$ as

$$
\begin{aligned}
& \mathrm{S}(x, y)=(\mathbf{I}-\mathbf{N}) x+\mathbf{M} y, \\
& \mathrm{~T}(x, y)=(\mathbf{I}-\mathbf{N}) y-\mathbf{M} x
\end{aligned}
$$


for all $x, y \in H$. Suppose that equations (24) and (25) are linearly dependent, then there exists $d \in \mathbb{R} \backslash\{0\}$ such that $\mathrm{S}(x, y)=d \mathrm{~T}(x, y)$ for all $x, y \in H$ or

$$
(\mathbf{I}-\mathbf{N}) x+\mathbf{M} y=d((\mathbf{I}-\mathbf{N}) y-\mathbf{M} x), \quad \forall x, y \in H .
$$

It follows from this that

$$
\begin{aligned}
& (\mathbf{I}-\mathbf{N}) x=-d \mathbf{M} x, \\
& \mathbf{M} y=d(\mathbf{I}-\mathbf{N}) y
\end{aligned}
$$

for all $x, y \in H$. Note that the Robin problem is uniquely solvable subject to $\frac{\alpha(t)}{\beta(t)}>0$. Therefore the system of integral equations

$$
\begin{aligned}
& (\mathbf{I}-\mathbf{N}) \mu=-\mathbf{M} \gamma, \\
& (\mathbf{I}-\mathbf{N}) \gamma=\mathbf{M} \mu
\end{aligned}
$$

has the solution $(\mu, \gamma)$, where $\gamma(t)=\gamma_{1}(t)-\mu_{2}(t)+c_{1}$ and $\mu(t)=\gamma_{2}(t)+\mu_{1}(t)+c_{2}$. Equations (26) and (27) are true for $x=\mu$ and $y=\gamma$, i.e.,

$$
\begin{aligned}
& (\mathbf{I}-\mathbf{N}) \mu=-d \mathbf{M} \mu, \\
& \mathbf{M} \gamma=d(\mathbf{I}-\mathbf{N}) \gamma .
\end{aligned}
$$

Using (28) and (29), equations (30) and (31) become

$$
\begin{aligned}
& \mathbf{M} \gamma=d \mathbf{M} \mu, \\
& -(\mathbf{I}-\mathbf{N}) \mu=d(\mathbf{I}-\mathbf{N}) \gamma .
\end{aligned}
$$

Multiplying both sides of (33) by $(\mathbf{I}-\mathbf{N})^{-1}$, we get

$$
-\mu=d \gamma
$$

Then from (32), we have

$$
\mathbf{M} \gamma=-d^{2} \mathbf{M} \gamma
$$

Since (28) and (29) has non-trivial solutions $(\mu, \gamma)$ and $(\mathbf{I}-\mathbf{N})$ is invertible, $\mathbf{M} \gamma \neq 0$. Hence by (34)

$$
d^{2}=-1
$$

It contradicts $d \in \mathbb{R}$. The theorem is proven.

Equations (22) and (23) imply

$$
\begin{gathered}
(\mathbf{I}-\mathbf{N}) \mu_{1}(t)-\mathbf{M} \mu_{2}(t)+\mathbf{M} c_{1}+(\mathbf{I}-\mathbf{N}) c_{2} \\
=-\mathbf{M} \gamma_{1}(t)-(\mathbf{I}-\mathbf{N}) \gamma_{2}(t), \quad t \in J,
\end{gathered}
$$




$$
\begin{gathered}
-\mathbf{M} \mu_{1}(t)-(\mathbf{I}-\mathbf{N}) \mu_{2}(t)+(\mathbf{I}-\mathbf{N}) c_{1}-\mathbf{M} c_{2} \\
=-(\mathbf{I}-\mathbf{N}) \gamma_{1}(t)+\mathbf{M} \gamma_{2}(t), \quad t \in J .
\end{gathered}
$$

Applying the definitions of the integral operators $\mathbf{N}$ and $\mathbf{M}$, we get

$$
\begin{aligned}
& \mu_{1}(\tau)-\int_{J} N(\eta(\tau), \eta(t)) \mu_{1}(t) d t-\int_{J} M(\eta(\tau), \eta(t)) \mu_{2}(t) d t \\
& \quad+\int_{J} M(\eta(\tau), \eta(t)) c_{1} d t+\left(c_{2}-\int_{J} N(\eta(\tau), \eta(t)) c_{2} d t\right) \\
& =-\int_{J} M(\eta(\tau), \eta(t)) \gamma_{1}(t) d t-\left(\gamma_{2}(\tau)-\int_{J} N(\eta(\tau), \eta(t)) \gamma_{2}(t) d t\right), \quad \tau \in J, \\
& -\int_{J} M(\eta(\tau), \eta(t)) \mu_{1}(t) d t-\mu_{2}(\tau)+\int_{J} N(\eta(\tau), \eta(t)) \mu_{2}(t) d t \\
& \quad+\left(c_{1}-\int_{J} N(\eta(\tau), \eta(t)) c_{1} d t\right)-\int_{J} M(\eta(\tau), \eta(t)) c_{2} d t \\
& =-\left(\gamma_{1}(\tau)-\int_{J} N(\eta(\tau), \eta(t)) \gamma_{1}(t) d t\right)+\int_{J} M(\eta(\tau), \eta(t)) \gamma_{2}(t) d t, \quad \tau \in J .
\end{aligned}
$$

The system of integral equations (37) and (38) is in two unknown real functions $\mu_{1}(t), \mu_{2}(t)$ and two unknown real constants $c_{1}, c_{2}$. Furthermore, by the definitions of the functions $\mu_{1}(t), \mu_{2}(t)$ given in (17), (18), we have a condition in the form of a differential equation,

$$
\sin (\zeta(t)) \mu_{1}^{\prime}(t)-\cos (\zeta(t)) \mu_{2}^{\prime}(t)=0, \quad t \in J
$$

and the conditions

$$
\mu_{1}(0)=0, \quad \text { and } \quad \mu_{2}(0)=0 .
$$

For bounded region $\Omega$, since $\operatorname{Im} f(0)=0$, we have $\operatorname{Re}[g(0)]=0$. Applying the Cauchy integral formula for the bounded region $\Omega$ in Theorem 2 together with (19), (20), and (21), yields

$$
\begin{aligned}
g(0) & =\frac{1}{2 \pi \mathrm{i}} \int_{\Gamma} \frac{g(\eta)}{\eta} d \eta=\frac{1}{2 \pi \mathrm{i}} \int_{\Gamma} \frac{g(\eta(t))}{\eta(t)} \dot{\eta}(t) d t \\
& =\frac{1}{2 \pi \mathrm{i}} \int_{J} \frac{\left[\gamma_{1}(t)-\mu_{2}(t)+c_{1}+\mathrm{i}\left(\gamma_{2}(t)+\mu_{1}(t)+c_{2}\right)\right] \dot{\eta}(t)}{A(t) \eta(t)} d t, \quad t \in J .
\end{aligned}
$$

Thus $\operatorname{Re}[g(0)]=0$ implies that

$$
\int_{J}\left[\left(\gamma_{1}(t)-\mu_{2}(t)+c_{1}\right) \operatorname{Im} \frac{\dot{\eta}(t)}{A(t) \eta(t)}+\left(\gamma_{2}(t)+\mu_{1}(t)+c_{2}\right) \operatorname{Re} \frac{\dot{\eta}(t)}{A(t) \eta(t)}\right] d t=0, \quad t \in J
$$

which gives rise to the following condition for $\mu_{1}(t), \mu_{2}(t), c_{1}$, and $c_{2}$ :

$$
\begin{aligned}
& \int_{J} \operatorname{Re} \frac{\dot{\eta}(t)}{A(t) \eta(t)} \mu_{1}(t) d t-\int_{J} \operatorname{Im} \frac{\dot{\eta}(t)}{A(t) \eta(t)} \mu_{2}(t) d t+\int_{J} \operatorname{Im} \frac{\dot{\eta}(t)}{A(t) \eta_{0}(t)} c_{1} d t \\
& \quad+\int_{J} \operatorname{Re} \frac{\dot{\eta}(t)}{A(t) \eta(t)} c_{2} d t=-\int_{J} \operatorname{Im} \frac{\dot{\eta}(t)}{A(t) \eta(t)} \gamma_{1}(t) d t-\int_{J} \operatorname{Re} \frac{\dot{\eta}(t)}{A(t) \eta_{0}(t)} \gamma_{2}(t) d t .
\end{aligned}
$$


In summary to solve the Robin problem (1) on a bounded multiply connected regions, we solve for $\mu_{1}(t), \mu_{2}(t), c_{1}$, and $c_{2}$ from (37), (38), (40), and (41). Then we compute $g(\eta(t))$ from (14). Using the relation $g=-\mathrm{i} f$ we get $f(\eta(t))=\mathrm{i} g(\eta(t))$ and hence $u(\eta(t))=$ $\operatorname{Re}[f(\eta(t))]$. The interior values of $f$ can be computed via the Cauchy integral formula (9), which yields $u(z)=\operatorname{Re}[f(z)]$ for $z \in \Omega$.

For an unbounded region $\Omega$, since $f(\infty)=0$, we have $g(\infty)=0$. By using the Cauchy integral formula for the unbounded region $\Omega$ in Theorem 2, we have

$$
\frac{1}{2 \pi \mathrm{i}} \int_{\Gamma} \frac{g(\eta)}{\eta-z} d \eta= \begin{cases}g(z)-g(\infty), & z \in \Omega \\ -g(\infty), & z \notin \Omega\end{cases}
$$

Since $z=0 \notin \Omega$,

$$
\frac{1}{2 \pi \mathrm{i}} \int_{\Gamma} \frac{g(\eta)}{\eta} d \eta=-g(\infty)=0
$$

Applying (19), (20), and (21), we get

$$
\frac{1}{2 \pi \mathrm{i}} \int_{J} \frac{\gamma_{1}(t)-\mu_{2}(t)+c_{1}+\mathrm{i}\left(\gamma_{2}(t)+\mu_{1}(t)+c_{2}\right)}{A(t)} \frac{\dot{\eta}(t) d t}{\eta(t)}=0, \quad t \in J
$$

or, equivalently,

$$
\int_{J}\left[\gamma_{1}(t)-\mu_{2}(t)+c_{1}+\mathrm{i}\left(\gamma_{2}(t)+\mu_{1}(t)+c_{2}\right)\right]\left[\operatorname{Re} \frac{\dot{\eta}(t)}{A(t) \eta(t)}+\mathrm{i} \operatorname{Im} \frac{\dot{\eta}(t)}{A(t) \eta(t)}\right] d t=0
$$

which implies

$$
\begin{aligned}
& \int_{J}\left[\left(\gamma_{1}(t)-\mu_{2}(t)+c_{1}\right) \operatorname{Im} \frac{\dot{\eta}(t)}{A(t) \eta(t)}\right. \\
& \left.\quad+\left(\gamma_{2}(t)+\mu_{1}(t)+c_{2}\right) \operatorname{Re} \frac{\dot{\eta}(t)}{A(t) \eta(t)}\right] d t \\
& \quad-\mathrm{i} \int_{J}\left[\left(\gamma_{1}(t)-\mu_{2}(t)+c_{1}\right) \operatorname{Re} \frac{\dot{\eta}(t)}{A(t) \eta(t)}\right. \\
& \left.\quad-\left(\gamma_{2}(t)+\mu_{1}(t)+c_{2}\right) \operatorname{Im} \frac{\dot{\eta}(t)}{A(t) \eta(t)}\right] d t=0, \quad t \in J .
\end{aligned}
$$

The real part and imaginary part of equation (42), respectively, yield

$$
\int_{J}\left[\left(\gamma_{1}(t)-\mu_{2}(t)+c_{1}\right) \operatorname{Im} \frac{\dot{\eta}(t)}{A(t) \eta(t)}+\left(\gamma_{2}(t)+\mu_{1}(t)+c_{2}\right) \operatorname{Re} \frac{\dot{\eta}(t)}{A(t) \eta(t)}\right] d t=0, \quad t \in J
$$

and

$$
\int_{J}\left[\left(\gamma_{1}(t)-\mu_{2}(t)+c_{1}\right) \operatorname{Re} \frac{\dot{\eta}(t)}{A(t) \eta(t)}-\left(\gamma_{2}(t)+\mu_{1}(t)+c_{2}\right) \operatorname{Im} \frac{\dot{\eta}(t)}{A(t) \eta(t)}\right] d t=0, \quad t \in J
$$


which gives rise to the conditions

$$
\begin{aligned}
\int_{J} \operatorname{Re} & \frac{\dot{\eta}(t)}{A(t) \eta(t)} \mu_{1}(t) d t-\int_{J} \operatorname{Im} \frac{\dot{\eta}(t)}{A(t) \eta(t)} \mu_{2}(t) d t \\
& +\int_{J} \operatorname{Im} \frac{\dot{\eta}(t)}{A(t) \eta(t)} c_{1} d t+\int_{J} \operatorname{Re} \frac{\dot{\eta}(t)}{A(t) \eta(t)} c_{2} d t \\
= & -\int_{J} \operatorname{Im} \frac{\dot{\eta}(t)}{A(t) \eta(t)} \gamma_{1}(t) d t-\int_{J} \operatorname{Re} \frac{\dot{\eta}(t)}{A(t) \eta(t)} \gamma_{2}(t) d t, \quad t \in J,
\end{aligned}
$$

and

$$
\begin{aligned}
& -\int_{J} \operatorname{Im} \frac{\dot{\eta}(t)}{A(t) \eta(t)} \mu_{1}(t) d t-\int_{J} \operatorname{Re} \frac{\dot{\eta}(t)}{A(t) \eta(t)} \mu_{2}(t) d t \\
& \quad+\int_{J} \operatorname{Re} \frac{\dot{\eta}(t)}{A(t) \eta(t)} c_{1} d t-\int_{J} \operatorname{Im} \frac{\dot{\eta}(t)}{A(t) \eta(t)} c_{2} d t \\
& =-\int_{J} \operatorname{Re} \frac{\dot{\eta}(t)}{A(t) \eta(t)} \gamma_{1}(t) d t-\int_{J} \operatorname{Im} \frac{\dot{\eta}(t)}{A(t) \eta(t)} \gamma_{2}(t) d t, \quad t \in J .
\end{aligned}
$$

In summary to solve the Robin problem (1) on an unbounded multiply connected regions, we solve for $\mu_{1}(t), \mu_{2}(t), c_{1}$, and $c_{2}$ from (37), (38) (40), (43), and (44). Then we compute $g(\eta(t))$ from (14). Using the relation $g=-\mathrm{i} f$ we get $f(\eta(t))=\mathrm{i} g(\eta(t))$ and hence $f(\eta(t))=$ $u(\eta(t))+\mathrm{i} v(\eta(t))$. The exterior values of $f$ can be computed via the Cauchy integral formula (10), which yields $u(z)=\operatorname{Re}[f(z)]$ for $z \in \Omega$.

\section{Numerical implementation}

Since the functions $A(t)$ and $\eta(t)$ are $2 \pi$-periodic, the integral equations in (35) and (36) can be best discretized on an equidistant grid by the Nyström method with trapezoidal rule using $n$ equidistant nodes [19]. Since $\mathbf{M}=\mathbf{M}_{1}-\mathbf{K}$, the integrals involving the singular kernel $K(\tau, t)$ are discretized using the Wittich method [20]. Define the $n$ equidistant collocation points $t_{i}$ by

$$
t_{i}=\frac{2 \pi(i-1)}{n}, \quad i=1,2,3, \ldots, n
$$

Discretizing the integral equations (37) and (38) on $J$, we obtain, respectively, the linear systems

$$
\begin{aligned}
\mu_{1}\left(t_{i}\right) & -\frac{2 \pi}{n} \sum_{j=1}^{n} N\left(\eta\left(t_{i}\right), \eta\left(t_{j}\right)\right) \mu_{1}\left(t_{j}\right)+\sum_{j=1}^{n} K\left(t_{i}, t_{j}\right) \mu_{2}\left(t_{j}\right)-\frac{2 \pi}{n} \sum_{j=1}^{n} M_{1}\left(\eta\left(t_{i}\right), \eta\left(t_{j}\right)\right) \mu_{2}\left(t_{j}\right) \\
& -\sum_{j=1}^{n} K\left(t_{i}, t_{j}\right) c_{1}+\frac{2 \pi}{n} \sum_{j=1}^{n} M_{1}\left(\eta\left(t_{i}\right), \eta\left(t_{j}\right)\right) c_{1}+c_{2}-\frac{2 \pi}{n} \sum_{j=1}^{n} N\left(\eta\left(t_{i}\right), \eta\left(t_{j}\right)\right) c_{2} \\
= & -\left(\gamma_{2}\left(t_{i}\right)-\frac{2 \pi}{n} \sum_{j=1}^{n} N\left(\eta\left(t_{i}\right), \eta\left(t_{j}\right)\right) \gamma_{2}\left(t_{j}\right)\right) \\
& +\sum_{j=1}^{n} K\left(t_{i}, t_{j}\right) \gamma_{1}\left(t_{j}\right)-\frac{2 \pi}{n} \sum_{j=1}^{n} M_{1}\left(\eta\left(t_{i}\right), \eta\left(t_{j}\right)\right) \gamma_{1}\left(t_{j}\right)
\end{aligned}
$$


and

$$
\begin{aligned}
\sum_{j=1}^{n} K\left(t_{i}, t_{j}\right) \mu_{1}\left(t_{j}\right)-\frac{2 \pi}{n} \sum_{j=1}^{n} M_{1}\left(\eta\left(t_{i}\right), \eta\left(t_{j}\right)\right) \mu_{1}\left(t_{j}\right)-\mu_{2}\left(t_{i}\right) \\
+\frac{2 \pi}{n} \sum_{j=1}^{n} N\left(\eta\left(t_{i}\right), \eta\left(t_{j}\right)\right) \mu_{2}\left(t_{j}\right)+c_{1}-\frac{2 \pi}{n} \sum_{j=1}^{n} N\left(\eta\left(t_{i}\right), \eta\left(t_{j}\right)\right) c_{1} \\
+\sum_{j=1}^{n} K\left(t_{i}, t_{j}\right) c_{2}-\frac{2 \pi}{n} \sum_{j=1}^{n} M_{1}\left(\eta\left(t_{i}\right), \eta\left(t_{j}\right)\right) c_{2} \\
=-\gamma_{1}\left(t_{i}\right)+\frac{2 \pi}{n} \sum_{j=1}^{n} N\left(\eta\left(t_{i}\right), \eta\left(t_{j}\right)\right) \gamma_{1}\left(t_{j}\right)-\sum_{j=1}^{n} K\left(t_{i}, t_{j}\right) \gamma_{2}\left(t_{j}\right) \\
+\frac{2 \pi}{n} \sum_{j=1}^{n} M_{1}\left(\eta\left(t_{i}\right), \eta\left(t_{j}\right)\right) \gamma_{2}\left(t_{j}\right),
\end{aligned}
$$

where

$$
\begin{aligned}
& K\left(t_{i}, t_{j}\right)= \begin{cases}0, & \text { if } j-i \text { is even, } \\
\frac{2}{n} \cot \frac{(i-j) \pi}{n}, & \text { if } j-i \text { is odd, }\end{cases} \\
& N\left(\eta\left(t_{i}\right), \eta\left(t_{j}\right)\right)= \begin{cases}\frac{1}{\pi} \operatorname{Im}\left[\frac{A\left(t_{i}\right)}{A\left(t_{j}\right)} \frac{\dot{\eta}\left(t_{j}\right)}{\eta\left(t_{j}\right)-\eta_{1}\left(t_{i}\right)}\right], & \text { if } t_{i} \neq t_{j}, \\
\frac{1}{\pi} \operatorname{Im}\left[\frac{1}{2} \frac{\ddot{\eta}\left(t_{i}\right)}{\dot{\eta}\left(t_{i}\right)}-\frac{\dot{A}\left(t_{i}\right)}{A\left(t_{i}\right)}\right], & \text { if } t_{i}=t_{j},\end{cases}
\end{aligned}
$$

and

$$
M_{1}\left(\eta\left(t_{i}\right), \eta\left(t_{j}\right)\right)= \begin{cases}\frac{1}{\pi} \operatorname{Re}\left[\frac{A\left(t_{i}\right)}{A\left(t_{j}\right)} \frac{\dot{\eta}\left(t_{j}\right)}{\eta\left(t_{j}\right)-\eta\left(t_{i}\right)}\right]+\frac{1}{2 \pi} \cot \frac{t_{i}-t_{j}}{2}, & \text { if } t_{i} \neq t_{j}, \\ \frac{1}{\pi} \operatorname{Re}\left[\frac{1}{2} \frac{\ddot{\eta}\left(t_{i}\right)}{\dot{\eta}\left(t_{i}\right)}-\frac{\dot{A}\left(t_{i}\right)}{A\left(t_{i}\right)}\right], & \text { if } t_{i}=t_{j} .\end{cases}
$$

Hence, we obtain $2 m n$ equations in $2 m n+2 m$ variables $(m=1,2, \ldots)$ :

$$
\begin{aligned}
& \mu_{01}\left(t_{1}\right), \mu_{01}\left(t_{2}\right), \ldots, \mu_{01}\left(t_{n}\right), \ldots, \mu_{0 m}\left(t_{1}\right), \mu_{0 m}\left(t_{2}\right) \ldots, \mu_{0 m}\left(t_{n}\right), \\
& \mu_{11}\left(t_{1}\right), \mu_{11}\left(t_{2}\right), \ldots, \mu_{11}\left(t_{n}\right), \ldots, \mu_{1 m}\left(t_{1}\right), \mu_{1 m}\left(t_{2}\right) \ldots, \mu_{1 m}\left(t_{n}\right), \\
& c_{01}, \ldots c_{0 m}, c_{11}, \ldots, c_{1 m} .
\end{aligned}
$$

The condition (39) is discretized using a five-point central difference method [21] to obtain $m n$ equations. For $t_{i} \in J$, we obtain for $i=1$

$$
\begin{aligned}
& \sin \left(\zeta\left(t_{1}\right)\right)\left[-25 \mu_{1}\left(t_{1}\right)+48 \mu_{1}\left(t_{2}\right)-36 \mu_{1}\left(t_{3}\right)+16 \mu_{1}\left(t_{4}\right)-3 \mu_{1}\left(t_{5}\right)\right] \\
& \quad-\cos \left(\zeta\left(t_{1}\right)\right)\left[-25 \mu_{2}\left(t_{1}\right)+48 \mu_{2}\left(t_{2}\right)-36 \mu_{2}\left(t_{3}\right)+16 \mu_{2}\left(t_{4}\right)-3 \mu_{2}\left(t_{5}\right)\right]=0 .
\end{aligned}
$$

For $i=2$, we have

$$
\begin{aligned}
& \sin \left(\zeta\left(t_{2}\right)\right)\left[-3 \mu_{1}\left(t_{1}\right)-10 \mu_{1}\left(t_{2}\right)+18 \mu_{1}\left(t_{3}\right)-6 \mu_{1}\left(t_{4}\right)+\mu_{1}\left(t_{5}\right)\right] \\
& \quad-\cos \left(\zeta\left(t_{2}\right)\right)\left[-3 \mu_{2}\left(t_{1}\right)-10 \mu_{2}\left(t_{2}\right)+18 \mu_{2}\left(t_{3}\right)-6 \mu_{2}\left(t_{4}\right)+\mu_{2}\left(t_{5}\right)\right]=0 .
\end{aligned}
$$


For $i=3, \ldots, n-2$, we have

$$
\begin{aligned}
& \sin \left(\zeta\left(t_{i}\right)\right)\left[\mu_{1}\left(t_{i-2}\right)-8 \mu_{1}\left(t_{i-1}\right)+8 \mu_{1}\left(t_{i+1}\right)-\mu_{1}\left(t_{i+2}\right)\right] \\
& \quad-\cos \left(\zeta\left(t_{i}\right)\right)\left[\mu_{2}\left(t_{i-2}\right)-8 \mu_{2}\left(t_{i-1}\right)+8 \mu_{2}\left(t_{i+1}\right)-\mu_{2}\left(t_{i+2}\right)\right]=0 .
\end{aligned}
$$

For $i=n-1$, we have

$$
\begin{aligned}
& \sin \left(\zeta\left(t_{n-1}\right)\right)\left[-\mu_{1}\left(t_{n-4}\right)+6 \mu_{1}\left(t_{n-3}\right)-18 \mu_{1}\left(t_{n-2}\right)+10 \mu_{1}\left(t_{n-1}\right)+3 \mu_{1}\left(t_{n}\right)\right] \\
& \quad-\cos \left(\zeta\left(t_{n-1}\right)\right)\left[-\mu_{2}\left(t_{n-4}\right)+6 \mu_{2}\left(t_{n-3}\right)-18 \mu_{2}\left(t_{n-2}\right)+10 \mu_{2}\left(t_{n-1}\right)+3 \mu_{2}\left(t_{n}\right)\right]=0 .
\end{aligned}
$$

For $i=n$, we have

$$
\begin{aligned}
& \sin \left(\zeta\left(t_{n}\right)\right)\left[3 \mu_{1}\left(t_{n-4}\right)-16 \mu_{1}\left(t_{n-3}\right)+36 \mu_{1}\left(t_{n-2}\right)-48 \mu_{1}\left(t_{n-1}\right)+25 \mu_{1}\left(t_{n}\right)\right] \\
& \quad-\cos \left(\zeta\left(t_{n}\right)\right)\left[3 \mu_{2}\left(t_{n-4}\right)-16 \mu_{2}\left(t_{n-3}\right)+36 \mu_{2}\left(t_{n-2}\right)-48 \mu_{2}\left(t_{n-1}\right)+25 \mu_{2}\left(t_{n}\right)\right]=0 .
\end{aligned}
$$

We now have $3 m n$ equations in $2 m n+2 m$ in unknowns (45). For the Robin problem on a bounded multiply connected region, discretizing condition (41) gives

$$
\begin{aligned}
\sum_{j=1}^{n} \operatorname{Re} & {\left[\frac{\dot{\eta}\left(t_{j}\right)}{A\left(t_{j}\right) \eta\left(t_{j}\right)}\right] \mu_{1}\left(t_{j}\right)-\sum_{j=1}^{n} \operatorname{Im}\left[\frac{\dot{\eta}\left(t_{j}\right)}{A\left(t_{j}\right) \eta\left(t_{j}\right)}\right] \mu_{2}\left(t_{j}\right) } \\
& +\sum_{j=1}^{n} \operatorname{Im}\left[\frac{\dot{\eta}\left(t_{j}\right)}{A\left(t_{j}\right) \eta\left(t_{j}\right)}\right] c_{1}+\sum_{j=1}^{n} \operatorname{Re}\left[\frac{\dot{\eta}\left(t_{j}\right)}{A\left(t_{j}\right) \eta\left(t_{j}\right)}\right] c_{2} \\
= & -\sum_{j=1}^{n} \operatorname{Im}\left[\frac{\dot{\eta}\left(t_{j}\right)}{A\left(t_{j}\right) \eta\left(t_{j}\right)}\right] \gamma_{1}\left(t_{j}\right)-\sum_{j=1}^{n} \operatorname{Re}\left[\frac{\dot{\eta}\left(t_{j}\right)}{A\left(t_{j}\right) \eta\left(t_{j}\right)}\right] \gamma_{2}\left(t_{j}\right) .
\end{aligned}
$$

For the Robin problem on an unbounded multiply connected region, discretizing conditions (43) and (44), we obtain

$$
\begin{aligned}
\sum_{j=1}^{n} \operatorname{Re}\left[\frac{\dot{\eta}\left(t_{j}\right)}{A\left(t_{j}\right) \eta\left(t_{j}\right)}\right] \mu_{1}\left(t_{j}\right)-\sum_{j=1}^{n} \operatorname{Im}\left[\frac{\dot{\eta}\left(t_{j}\right)}{A\left(t_{j}\right) \eta\left(t_{j}\right)}\right] \mu_{2}\left(t_{j}\right) \\
+\sum_{j=1}^{n} \operatorname{Im}\left[\frac{\dot{\eta}\left(t_{j}\right)}{A\left(t_{j}\right) \eta\left(t_{j}\right)}\right] c_{1}+\sum_{j=1}^{n} \operatorname{Re}\left[\frac{\dot{\eta}\left(t_{j}\right)}{A\left(t_{j}\right) \eta\left(t_{j}\right)}\right] c_{2} \\
=-\sum_{j=1}^{n} \operatorname{Im}\left[\frac{\dot{\eta}\left(t_{j}\right)}{A\left(t_{j}\right) \eta\left(t_{j}\right)}\right] \gamma_{1}\left(t_{j}\right)-\sum_{j=1}^{n} \operatorname{Re}\left[\frac{\dot{\eta}\left(t_{j}\right)}{A\left(t_{j}\right) \eta\left(t_{j}\right)}\right] \gamma_{2}\left(t_{j}\right), \\
-\sum_{j=1}^{n} \operatorname{Im}\left[\frac{\dot{\eta}\left(t_{j}\right)}{A\left(t_{j}\right) \eta\left(t_{j}\right)}\right] \mu_{1}\left(t_{j}\right)-\sum_{j=1}^{n} \operatorname{Re}\left[\frac{\dot{\eta}\left(t_{j}\right)}{A\left(t_{j}\right) \eta\left(t_{j}\right)}\right] \mu_{2}\left(t_{j}\right) \\
+\sum_{j=1}^{n} \operatorname{Re}\left[\frac{\dot{\eta}\left(t_{j}\right)}{A\left(t_{j}\right) \eta\left(t_{j}\right)}\right] c_{1}-\sum_{j=1}^{n} \operatorname{Im}\left[\frac{\dot{\eta}\left(t_{j}\right)}{A\left(t_{j}\right) \eta\left(t_{j}\right)}\right] c_{2} \\
=-\sum_{j=1}^{n} \operatorname{Re}\left[\frac{\dot{\eta}\left(t_{j}\right)}{A\left(t_{j}\right) \eta\left(t_{j}\right)}\right] \gamma_{1}\left(t_{j}\right)-\sum_{j=1}^{n} \operatorname{Im}\left[\frac{\dot{\eta}\left(t_{j}\right)}{A\left(t_{j}\right) \eta\left(t_{j}\right)}\right] \gamma_{2}\left(t_{j}\right) .
\end{aligned}
$$


Hence, for the Robin problem in a bounded multiply connected region we obtain ( $3 m n)$ by a $(2 m n+2 m)$ linear system, including the $2 m$ condition in (40) giving a $(3 m n+2 m)$ by $(2 m n+2 m)$ linear system. Finally, adding the equation obtained from (41) makes a $(3 m n+2 m+1)$ by $(2 m n+2 m)$ over-determined linear system which is full ranked.

For the Robin problem in an unbounded multiply connected region we obtain a ( $3 m n)$ by $(2 m n+2 m)$ linear system, including the $2 m$ condition in (40) giving a $(3 m n+2 m)$ by $(2 m n+2 m)$ linear system. Finally, adding the two equations obtained from (43) and (44) makes a $(3 m n+2 m+2)$ by $(2 m n+2 m)$ over-determined linear system which is full ranked.

In both cases, the obtained over-determined systems are solved using the MATLAB's $\backslash$ operator that makes use the QR factorization with a column pivoting method.

From the computed solutions $\mu_{1}(t), \mu_{2}(t), \gamma_{1}(t), \gamma_{2}(t), c_{1}$, and $c_{2}$, the approximate boundary values of the analytic function $f_{n}(\eta(t))$ are calculated using the formula

$$
f_{n}(\eta(t))=\frac{-\left(\gamma_{2}(t)+\mu_{1}(t)+c_{2}\right)+\mathrm{i}\left(\gamma_{1}(t)-\mu_{2}(t)+c_{1}\right)}{e^{\mathrm{i} \zeta(t)}} .
$$

The approximate interior values of the function $f(z)$ are calculated via the Cauchy integral formula in the form of

$$
f(z)= \begin{cases}\frac{\frac{1}{2 \pi \mathrm{i}} \int_{J} \frac{f(\eta)}{\eta-z} d \eta}{\frac{1}{2 \pi \mathrm{i}} \int_{J} \frac{d \eta}{\eta-z}} & {[\text { Bounded region } \Omega],} \\ \frac{f(\infty)+\frac{1}{2 \pi \mathrm{i}} \int_{J} \frac{f(\eta)}{\eta-z} d \eta}{1+\frac{1}{2 \pi \mathrm{i}} \int_{J} \frac{d \eta}{\eta-z}} & {[\text { Unbounded region } \Omega] .}\end{cases}
$$

Here $f(\infty)=0$ for unbounded region $\Omega$. The formula in (46) has the advantage that the denominators in this formula compensate for the error in the numerators [22, 23]. The integrals in (46) are approximated by the trapezoidal rule. The respective discretization formula for bounded and unbounded regions are

$$
f_{n}(z)= \begin{cases}\frac{\sum_{i=1}^{n} \frac{f_{n}\left(\eta\left(t_{i}\right)\right) \dot{\eta}\left(t_{i}\right)}{\eta\left(t_{i}\right)-z}}{\sum_{i=1}^{n} \frac{\dot{\eta}\left(t_{i}\right)}{\eta\left(t_{i}\right)-z}} & \text { [Bounded region } \Omega], \\ \frac{\sum_{i=1}^{n} \frac{\left.f_{n}\left(\eta \eta t_{i}\right)\right)\left(t_{i}\right)}{\eta\left(t_{i}\right)-z}}{\operatorname{in}+\sum_{i=1}^{n} \frac{\dot{\eta}\left(t_{i}\right)}{\eta\left(t_{i}\right)-z}} & \text { [Unbounded region } \Omega] .\end{cases}
$$

\section{Numerical examples}

We consider some examples of solving Robin problem with Robin boundary condition (1) in bounded and unbounded multiply connected regions.

Example 1 Consider a bounded triply connected region $\Omega$ bounded by

$$
\begin{array}{ll}
\Gamma_{0}: & \eta_{0}(t)=1.5 \cos t+0.6 \mathrm{i} \sin t, \\
\Gamma_{1}: & \eta_{1}(t)=0.7+0.3 e^{-\mathrm{i} t}, \\
\Gamma_{2}: & \eta_{2}(t)=-0.7+0.3 e^{-\mathrm{i} t}, \quad 0 \leq t \leq 2 \pi .
\end{array}
$$

For $\alpha(t), \beta(t)$, and $A(t)$ in (1), we choose

$$
\alpha(t)= \begin{cases}10-10 \sin t, & t \in J_{0}, \\ 2-2 \sin t, & t \in J_{1}, \\ 2 \cos t-\sin 2 t, & t \in J_{2},\end{cases}
$$


Table 1 The errors $\left\|u(\eta(t))-u_{n}(\eta(t))\right\|_{\infty}$ on boundary $\Gamma$ for Example 1

\begin{tabular}{rl}
\hline $\boldsymbol{n}$ & $\boldsymbol{\| u ( \eta ( t ) ) - \boldsymbol { u } _ { \boldsymbol { n } } ( \boldsymbol { \eta } ( \boldsymbol { t } ) ) \| _ { \infty }}$ \\
\hline 32 & $1.23(-2)$ \\
64 & $2.88(-4)$ \\
128 & $2.28(-5)$ \\
256 & $1.61(-6)$ \\
512 & $1.07(-7)$ \\
1,024 & $7.11(-9)$ \\
\hline
\end{tabular}

Table 2 Absolute errors $\left|f(z)-f_{n}(z)\right|$ at some selected points on $\Omega$ for Example 1

\begin{tabular}{rllll}
\hline $\boldsymbol{n}$ & $\mathbf{- 1 . 2 5 + 0 . 2 i}$ & $\mathbf{- 0 . 5 + 0 . 4 i}$ & $\mathbf{0 . 5 + 0 . 4 i}$ & $\mathbf{1 . 3 - 0 . 1 i}$ \\
\hline 32 & $2.10(-3)$ & $8.0(-4)$ & $8.0(-4)$ & $1.30(-3)$ \\
64 & $2.98(-5)$ & $6.11(-5)$ & $6.04(-5)$ & $3.31(-5)$ \\
128 & $1.80(-6)$ & $7.68(-6)$ & $6.80(-6)$ & $1.61(-6)$ \\
256 & $1.99(-7)$ & $5.79(-7)$ & $5.10(-7)$ & $8.08(-8)$ \\
512 & $1.57(-8)$ & $5.13(-8)$ & $3.53(-8)$ & $4.48(-9)$ \\
1,024 & $1.15(-9)$ & $3.32(-9)$ & $2.39(-9)$ & $3.08(-10)$ \\
\hline
\end{tabular}

and

$$
\beta(t)= \begin{cases}\left(3 \sqrt{21 \sin ^{2} t+4}\right), & t \in J_{0} \\ 0.6, & t \in J_{1}, \\ 0.6 \cos t, & t \in J_{2}\end{cases}
$$

The function $l(t)$ in (1) is obtained by choosing an exact solution $u(z)=\operatorname{Re}[f(z)]$, where $f(z)=\cos (z)-2$. This yields the exact values $c_{01}=0, c_{02}=1.9293, c_{11}=0, c_{12}=1.4597$, $c_{21}=0$, and $c_{22}=1.0789$. For this example, $A(t)=e^{\mathrm{i}(t+\cos t)}, t \in J$. The integrals in (15) and (16) are calculated by the Gauss-Legendre rule with 256 nodes.

Table 1 lists the maximum error norms $\left\|u(\eta(t))-u_{n}(\eta(t))\right\|_{\infty}$, where $n$ is the number of nodes and $u_{n}(\eta(t))$ is the numerical approximation of $u(\eta(t))$ based on our method. The errors $\left\|f(z)-f_{n}(z)\right\|$ at some selected points are listed in Table 2 . The absolute errors $\left|u(z)-u_{n}(z)\right|$ for selected points in the entire domain are plotted in Figure 3. Figure 4 shows the surface plot of $u_{n}(z)$ with $n=1,024$.

Example 2 Consider an unbounded triply connected region $\Omega$ with boundaries

$$
\begin{array}{ll}
\Gamma_{1}: & \eta_{1}(t)=0.5 \cos t-0.3 \mathrm{i} \sin t \\
\Gamma_{2}: & \eta_{2}(t)=-1+(0.2+0.04 \cos 4 t) e^{-\mathrm{i} t} \\
\Gamma_{3}: & \eta_{3}(t)=1+(0.2+0.04 \cos 4 t) e^{-\mathrm{i} t}, \quad 0 \leq t \leq 2 \pi
\end{array}
$$

For $\alpha(t)$ and $\beta(t)$ in (1), we choose

$$
\alpha(t)= \begin{cases}10+20 \cos t, & t \in J_{1}, \\ 1+2 \cos t, & t \in J_{2} \\ 1+2 \cos t, & t \in J_{3}\end{cases}
$$




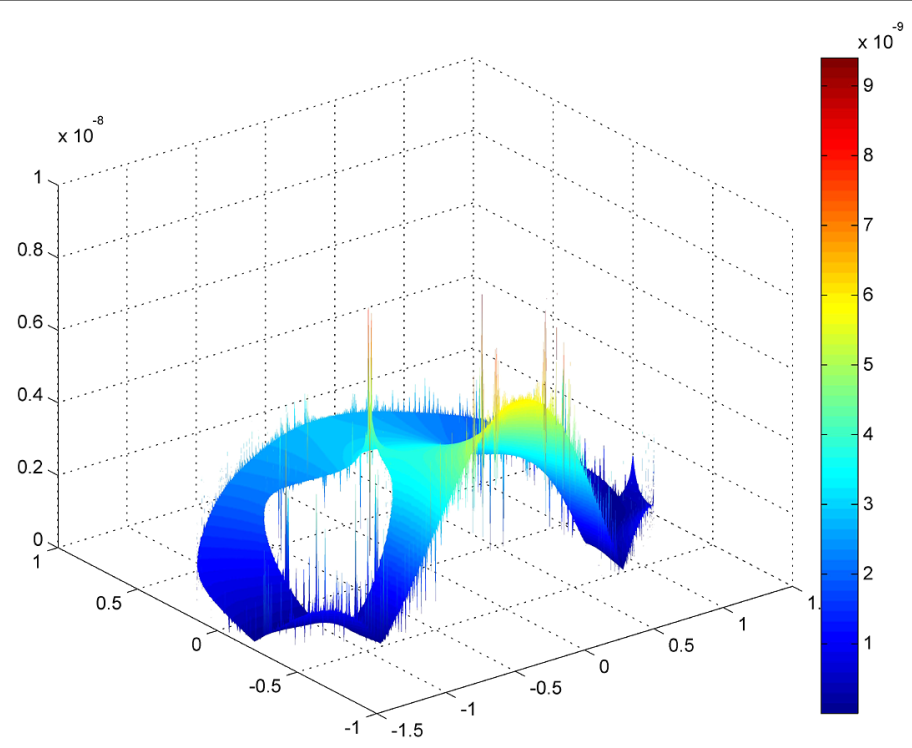

Figure 3 The absolute errors $\left|u(z)-u_{n}(z)\right|$ for the entire domain with $n=1,024$, for Example 1 .

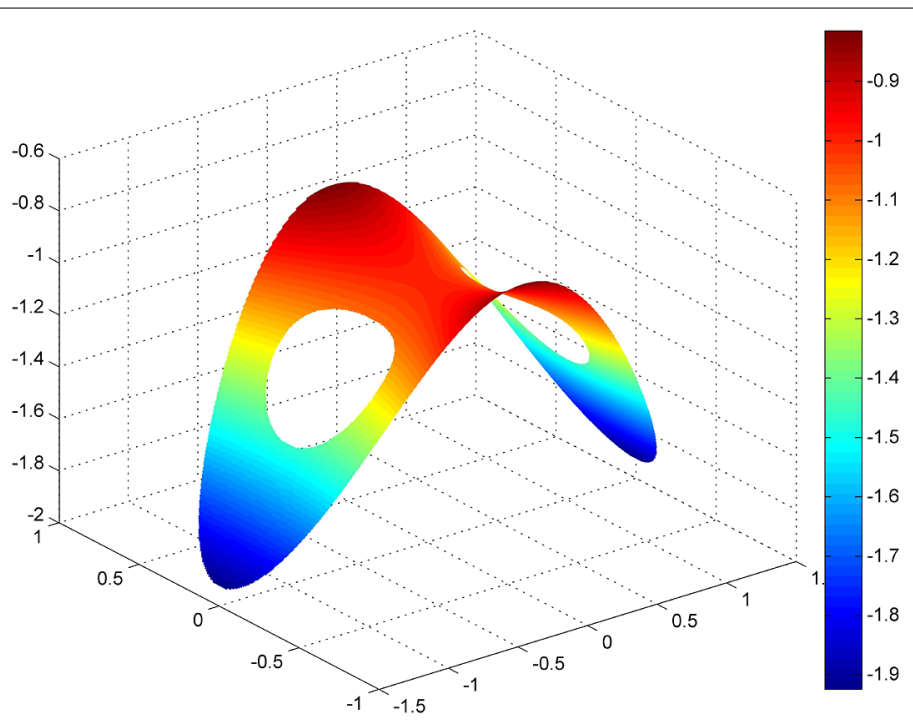

Figure 4 The surface plot of $u_{n}(z)$ for Example 1 with $n=1,024$.

and

$$
\beta(t)= \begin{cases}\left(\sqrt{25-16 \cos ^{2} t},\right. & t \in J_{1}, \\ \left|\left(\frac{e^{-i t} \cos 4 t}{25}+\frac{1}{5}\right) \mathrm{i}+\frac{4 \sin 4 t e^{-\mathrm{i} t}}{25}\right|, & t \in J_{2}, \\ \left(\left(6 2 5 \left(\left(\frac{\cos 5 t}{10}-\frac{3 \cos 3 t}{50}+\frac{\cos t}{5}\right)^{2}\right.\right.\right. & \\ \left.\left.\left.\quad+\left(\frac{3 \sin 3 t}{50}+\frac{\sin 5 t}{10}+\frac{\sin t}{5}\right)^{2}\right)\right)^{1 / 2}\right) / 25, & t \in J_{3} .\end{cases}
$$

The function $l(t)$ in (1) is obtained by choosing an exact solution $u(z)=\operatorname{Re}[f(z)]$, where $f(z)=\frac{1}{z}$. This yields the exact values, $c_{11}=0, c_{12}=-2, c_{21}=0, c_{22}=1.3158, c_{31}=0$, and $c_{32}=-0.8065$. For this example, $A(t)=e^{\mathrm{i}(t+2 \sin t)}, t \in J$. The integrals in (15) and (16) are calculated by the Gauss-Legendre rule with 256 nodes. 
Table 3 The errors $\left\|u(\eta(t))-u_{n}(\eta(t))\right\|_{\infty}$ on boundary $\Gamma$ for Example 2

\begin{tabular}{rl}
\hline $\boldsymbol{n}$ & $\boldsymbol{\| u ( \eta ( t ) ) - \boldsymbol { u } _ { \boldsymbol { n } } ( \boldsymbol { \eta } ( \boldsymbol { t } ) ) \| _ { \infty }}$ \\
\hline 32 & $1.38(-2)$ \\
64 & $9.62(-4)$ \\
128 & $6.47(-5)$ \\
256 & $3.87(-6)$ \\
512 & $4.73(-7)$ \\
1,024 & $6.57(-8)$ \\
\hline
\end{tabular}

Table 4 Absolute errors $\left|f(z)-f_{n}(z)\right|$ at some selected points on $\Omega$ for Example 2

\begin{tabular}{rllll}
\hline $\boldsymbol{n}$ & $\mathbf{- 0 . 6 - 0 . 2 i}$ & $\mathbf{0 . 7 5}$ & $\mathbf{0 . 8}+\mathbf{0 . 4 i}$ & $\mathbf{0 . 7 + 0 . 2 i}$ \\
\hline 32 & $7.0(-4)$ & $4.0(-4)$ & $1.0(-3)$ & $1.2(-3)$ \\
64 & $1.47(-5)$ & $2.03(-5)$ & $8.64(-5)$ & $1.08(-4)$ \\
128 & $6.51(-7)$ & $1.87(-6)$ & $6.51(-6)$ & $7.95(-6)$ \\
256 & $2.48(-7)$ & $6.02(-7)$ & $4.56(-7)$ & $5.87(-7)$ \\
512 & $7.30(-8)$ & $1.22(-7)$ & $4.07(-8)$ & $7.27(-8)$ \\
1,024 & $1.29(-8)$ & $1.96(-8)$ & $5.40(-9)$ & $1.11(-8)$ \\
\hline
\end{tabular}

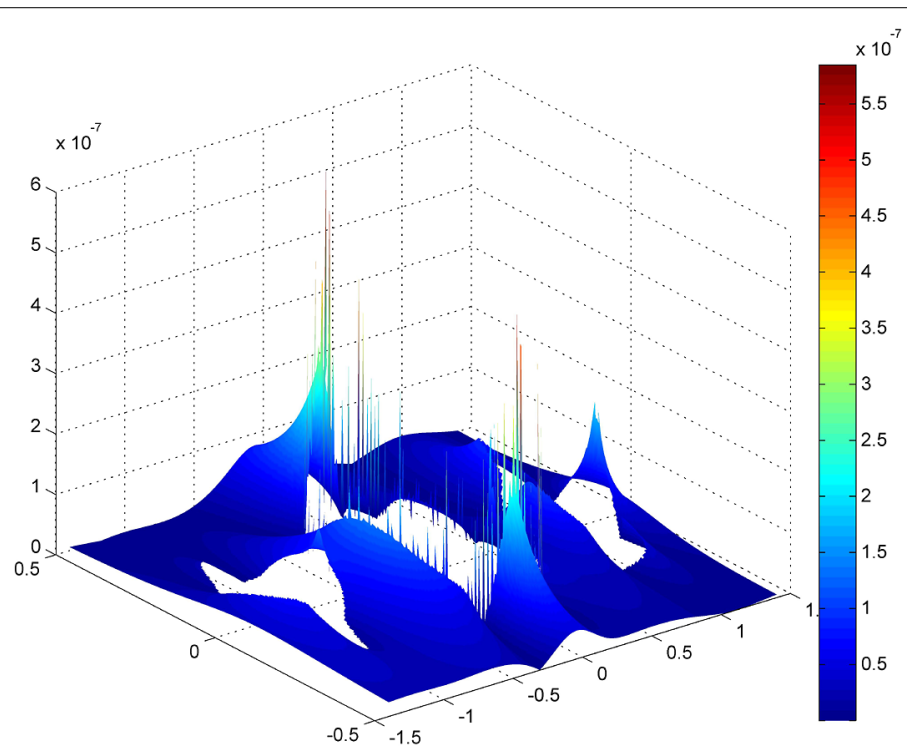

Figure 5 The absolute error $\left|u(z)-u_{n}(z)\right|$ for the entire domain with $n=512$, for Example 2 .

Table 3 lists the maximum error norms $\left\|u(\eta(t))-u_{n}(\eta(t))\right\|_{\infty}$, where $n$ is the number of nodes and $u_{n}(\eta(t))$ is the numerical approximation of $u(\eta(t))$ based on our method. The errors $\left\|f(z)-f_{n}(z)\right\|$ at some selected points are listed in Table 4 . The absolute errors $\left|u(z)-u_{n}(z)\right|$ for selected points in the entire domain are plotted in Figure 5. Figure 6 shows the surface plot of $u_{n}(z)$ with $n=512$.

Example 3 Consider a bounded 4-multiply connected region $\Omega$ with boundaries

$$
\begin{array}{ll}
\Gamma_{0}: & \eta_{0}(t)=1.5 \cos t+\mathrm{i} \sin t, \\
\Gamma_{1}: & \eta_{1}(t)=-0.5+0.3 \mathrm{i}+0.3 e^{-\mathrm{i} t}, \\
\Gamma_{2}: & \eta_{2}(t)=0.5+0.3 \mathrm{i}+0.3 e^{-\mathrm{i} t}, \\
\Gamma_{3}: & \eta_{3}(t)=-0.35 \mathrm{i}+0.3 e^{-\mathrm{i} t}, \quad 0 \leq t \leq 2 \pi .
\end{array}
$$




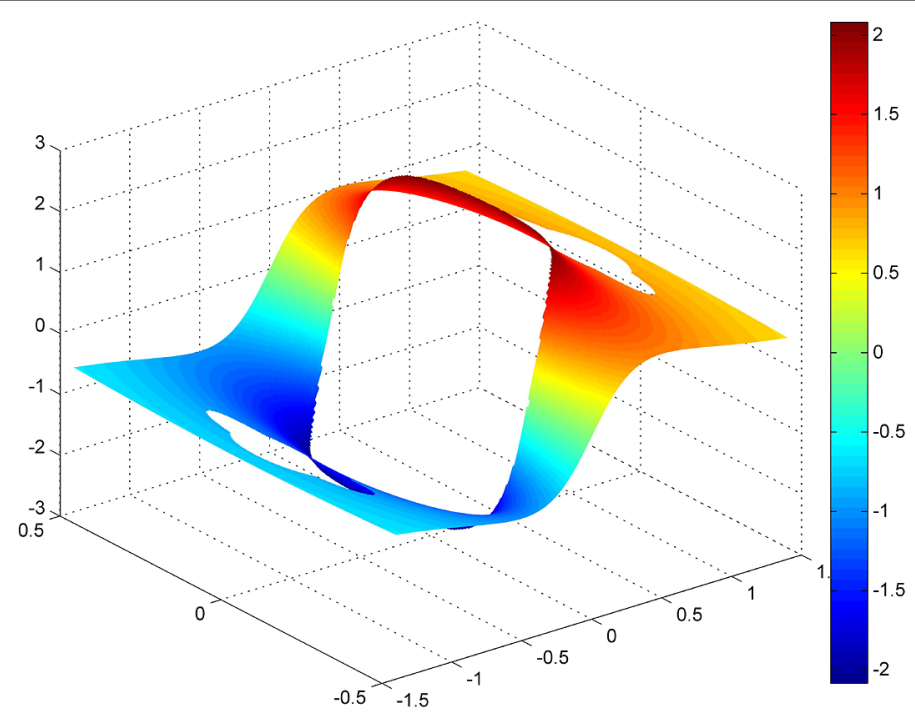

Figure 6 The surface plot of $u_{n}(z)$ for Example 2 with $n=512$.

For $\alpha(t)$ and $\beta(t)$ in (1), we choose

$$
\alpha(t)= \begin{cases}2-2 \cos t, & t \in J_{0}, \\ 1-\cos t+2 \sin t-\sin 2 t, & t \in J_{1}, \\ 1+\cos t-2 \cos ^{2} t, & t \in J_{2}, \\ 1-\cos t-2 \sin t+\sin 2 t, & t \in J_{3},\end{cases}
$$

and

$$
\beta(t)= \begin{cases}\sqrt{5 \sin ^{2}+4}, & t \in J_{0}, \\ 0.3+0.6 \sin t, & t \in J_{1}, \\ 0.3+0.6 \cos t, & t \in J_{2}, \\ 0.3-0.6 \sin t, & t \in J_{3} .\end{cases}
$$

The function $l(t)$ in (1) is obtained by choosing an exact solution $u(z)=\operatorname{Re}[f(z)]$, where $f(z)=z^{2}-2$. This yields the exact values, $c_{01}=0, c_{02}=-0.25, c_{11}=-0.12, c_{12}=2.05$, $c_{21}=0.48, c_{22}=1.45, c_{31}=-0.21$, and $c_{32}=2.0325$. For this example, $A(t)=e^{\mathrm{i}(t-\sin t)}$, $t \in J$. The integrals in (15) and (16) are calculated by the Gauss-Legendre rule with 256 nodes.

Table 5 lists the maximum error norms $\left\|u(\eta(t))-u_{n}(\eta(t))\right\|_{\infty}$, where $n$ is the number of nodes and $u_{n}(\eta(t))$ is the numerical approximation of $u(\eta(t))$ based on our method. The errors $\left\|f(z)-f_{n}(z)\right\|$ at some selected points are listed in Table 6 . The absolute errors $\left|u(z)-u_{n}(z)\right|$ for selected points in the entire domain are plotted in Figure 7 . Figure 8 shows the surface plot of $u_{n}(z)$ with $n=512$.

Example 4 Consider an unbounded 4-multiply connected region $\Omega$ with boundaries

$$
\begin{array}{ll}
\Gamma_{1}: & \eta_{1}(t)=0.95+(0.2+0.04 \cos (4 t)) e^{-\mathrm{i} t}, \\
\Gamma_{2}: & \eta_{2}(t)=0.2 e^{-\mathrm{i} t}
\end{array}
$$


Table 5 The errors $\left\|u(\eta(t))-u_{n}(\eta(t))\right\|_{\infty}$ on boundary $\Gamma$ for Example 3

\begin{tabular}{rl}
\hline $\boldsymbol{n}$ & $\boldsymbol{\| \boldsymbol { u } ( \boldsymbol { \eta } ( \boldsymbol { t } ) \boldsymbol { ) } - \boldsymbol { u } _ { \boldsymbol { n } } ( \boldsymbol { \eta } ( \boldsymbol { t } ) ) \| _ { \infty }}$ \\
\hline 32 & $1.69(-2)$ \\
64 & $8.61(-4)$ \\
128 & $4.98(-5)$ \\
256 & $3.11(-6)$ \\
512 & $1.99(-7)$ \\
1,024 & $1.32(-8)$ \\
\hline
\end{tabular}

Table 6 The absolute errors $\left|f(z)-f_{n}(z)\right|$ at some selected points on $\Omega$ for Example 3

\begin{tabular}{rllll}
\hline $\boldsymbol{n}$ & $\mathbf{- 1 - 0 . 2 i}$ & $-\mathbf{0 . 5}-\mathbf{0 . 6 i}$ & $\mathbf{0 . 2}+\mathbf{0 . 6 i}$ & $\mathbf{1 . 2 - 0 . 3 i}$ \\
\hline 32 & $8.0(-4)$ & $4.5(-3)$ & $3.4(-3)$ & $2.4(-3)$ \\
64 & $9.10(-5)$ & $2.24(-4)$ & $1.48(-4)$ & $1.10(-4)$ \\
128 & $6.68(-6)$ & $1.30(-5)$ & $8.13(-6)$ & $6.35(-6)$ \\
256 & $4.05(-7)$ & $8.47(-7)$ & $5.16(-7)$ & $4.07(-7)$ \\
512 & $2.33(-8)$ & $5.63(-8)$ & $3.46(-8)$ & $2.69(-8)$ \\
1,024 & $1.34(-9)$ & $3.74(-9)$ & $2.34(-9)$ & $1.78(-9)$ \\
\hline
\end{tabular}

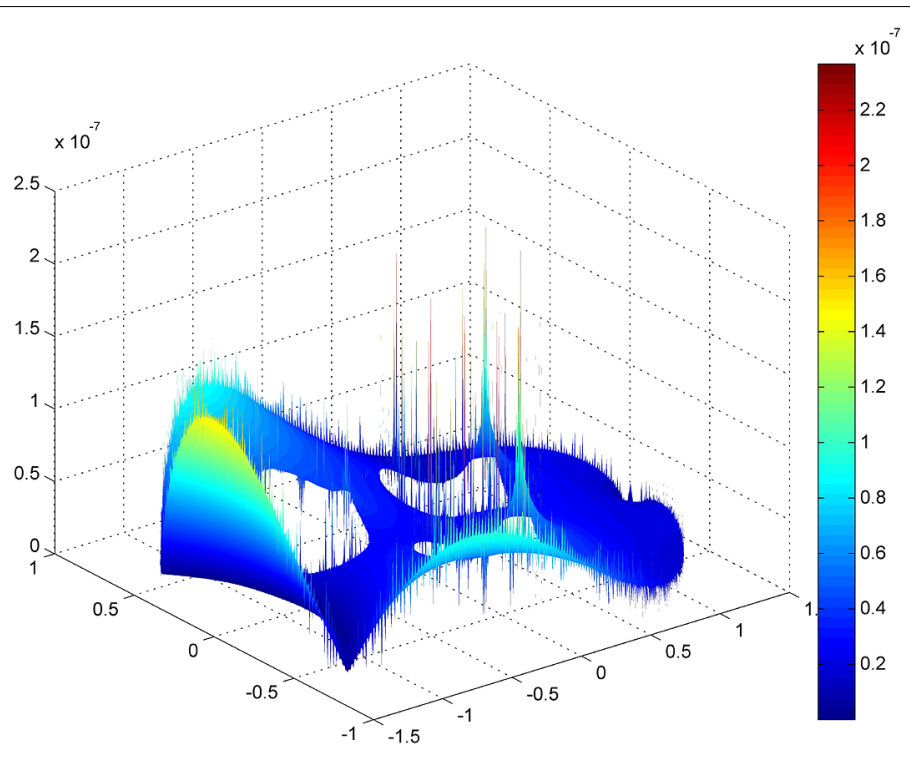

Figure 7 The absolute error $\left|u(z)-u_{n}(z)\right|$ for the entire domain with $n=512$, for Example 3 .

$$
\begin{array}{ll}
\Gamma_{3}: & \eta_{3}(t)=(0.5+0.45 \mathrm{i})+\left(0.2 e^{-\mathrm{i} t}\right), \\
\Gamma_{4}: & \eta_{4}(t)=(0.5-0.45 \mathrm{i})+(0.2 \cos (t)-0.1 \mathrm{i} \sin (t), \quad 0 \leq t \leq 2 \pi .
\end{array}
$$

For $\alpha(t)$ and $\beta(t)$ in (1), we choose

$$
\alpha(t)= \begin{cases}25-25 \sin t, & t \in J_{1}, \\ 1-\sin t+2 \cos t-\sin 2 t, & t \in J_{2}, \\ 3(1-\sin t-2 \cos t+\sin 2 t), & t \in J_{3}, \\ 10-10 \sin t, & t \in J_{4},\end{cases}
$$




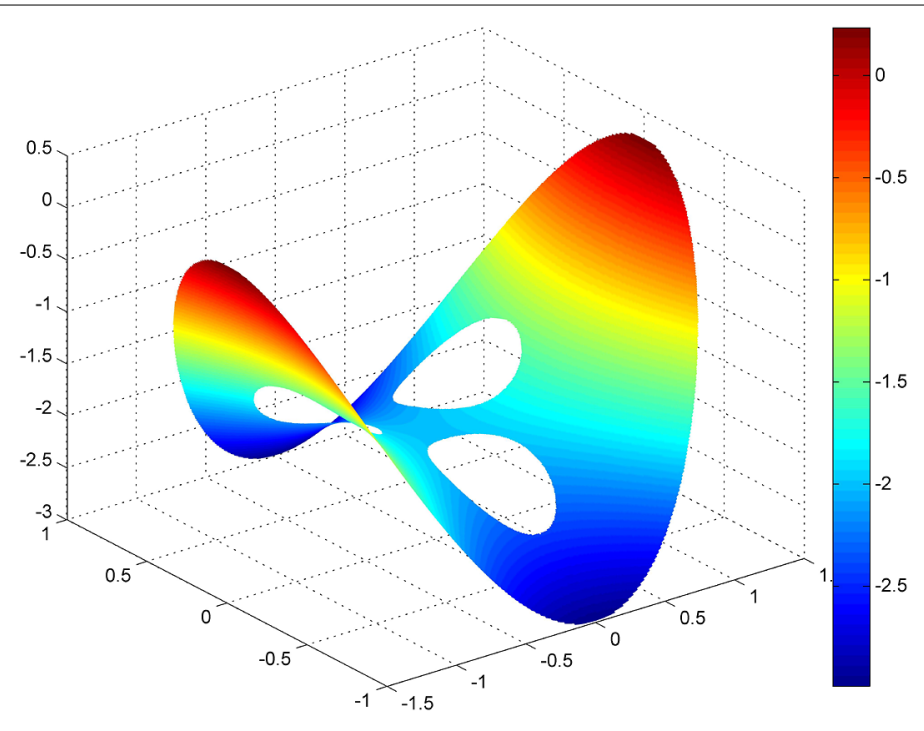

Figure 8 The surface plot of $u_{n}(z)$ for Example 3 with $n=512$.

Table 7 The errors $\left\|u(\eta(t))-u_{n}(\eta(t))\right\|_{\infty}$ on boundary $\Gamma$ for Example 4

\begin{tabular}{rl}
\hline \multicolumn{1}{l}{$\boldsymbol{n}$} & $\boldsymbol{\| \boldsymbol { u } ( \boldsymbol { \eta } ( \boldsymbol { t } ) \boldsymbol { ) } - \boldsymbol { u } _ { \boldsymbol { n } } ( \boldsymbol { \eta } ( \boldsymbol { t } ) ) \| _ { \infty }}$ \\
\hline 32 & $1.56(-2)$ \\
64 & $8.57(-4)$ \\
128 & $5.16(-5)$ \\
256 & $3.21(-6)$ \\
512 & $1.99(-7)$ \\
1,024 & $1.24(-8)$ \\
\hline
\end{tabular}

Table 8 The absolute errors $\left|f(z)-f_{n}(z)\right|$ at some selected points on $\Omega$ for Example 4

\begin{tabular}{rllll}
\hline $\boldsymbol{n}$ & $\mathbf{0 . 2}-\mathbf{0 . 4 i}$ & $\mathbf{0 . 3}-\mathbf{0 . 2 i}$ & $\mathbf{0 . 6}+\mathbf{0 . 2 i}$ & $\mathbf{1}+\mathbf{0 . 5 i}$ \\
\hline 32 & $7.0(-4)$ & $1.6(-3)$ & $1.3(-3)$ & $1.1(-3)$ \\
64 & $3.87(-5)$ & $1.02(-4)$ & $8.92(-5)$ & $37.07(-5)$ \\
128 & $2.04(-6)$ & $6.23(-6)$ & $5.86(-6)$ & $4.58(-6)$ \\
256 & $9.83(-8)$ & $3.66(-7)$ & $3.76(-7)$ & $2.97(-7)$ \\
512 & $4.34(-9)$ & $2.12(-8)$ & $2.39(-8)$ & $1.92(-8)$ \\
1,024 & $1.87(-10)$ & $1.22(-9)$ & $1.51(-9)$ & $1.24(-9)$ \\
\hline
\end{tabular}

and

$$
\beta(t)= \begin{cases}\left(\left(6 2 5 \left(\left(\frac{\cos 5 t}{10}-\frac{3 \cos 3 t}{50}+\frac{\cos t}{5}\right)^{2}\right.\right.\right. & \\ \left.\left.\left.\quad+\left(\frac{3 \sin 3 t}{50}+\frac{\sin 5 t}{10}+\frac{\sin t}{5}\right)^{2}\right)\right)^{1 / 2}\right), & t \in J_{1}, \\ 0.2+0.4 \cos t, & t \in J_{2}, \\ 0.6-1.2 \cos t, & t \in J_{3}, \\ \sqrt{3 \sin ^{2} t+1,} & t \in J_{4} .\end{cases}
$$

The function $l(t)$ in (1) is obtained by choosing an exact solution $u(z)=\operatorname{Re}[f(z)]$, where $f(z)=\frac{1}{z}$. This yields the exact values, $c_{11}=0, c_{12}=-0.8403, c_{21}=0, c_{22}=-5, c_{31}=-0.6498$, $c_{32}=-1.0108, c_{41}=0.6498$, and $c_{42}=-1.0108$. For this example, $A(t)=e^{\mathrm{i}(t+\cos t)}, t \in J$. The integrals in (15) and (16) are calculated by the Gauss-Legendre rule with 256 nodes. 


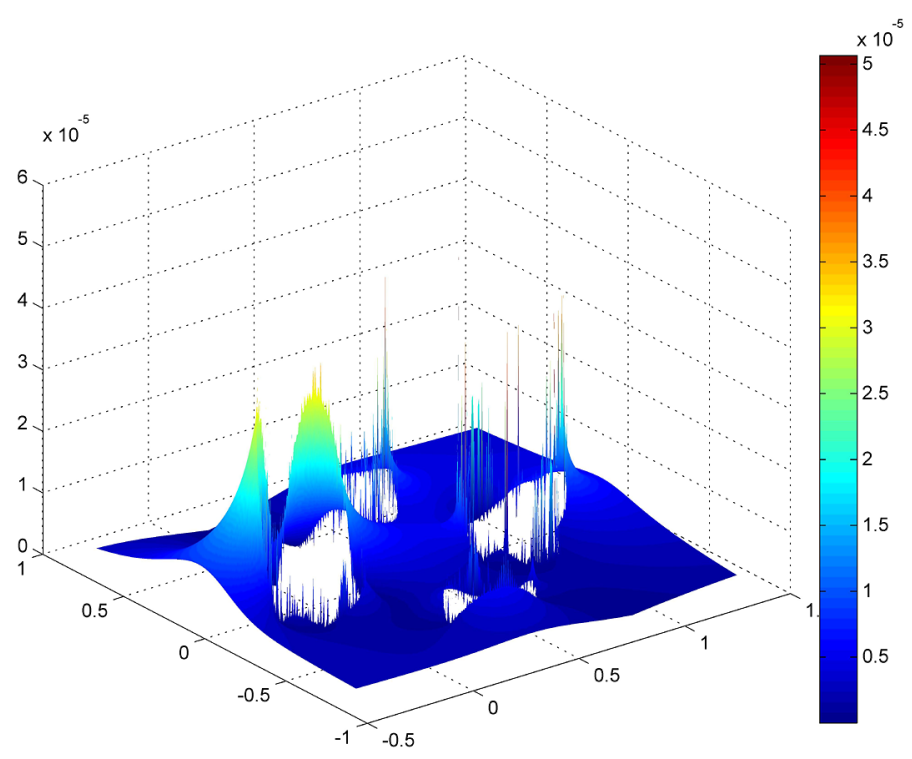

Figure 9 The absolute error $\left|u(z)-u_{n}(z)\right|$ for the entire domain with $n=128$, for Example 4 .

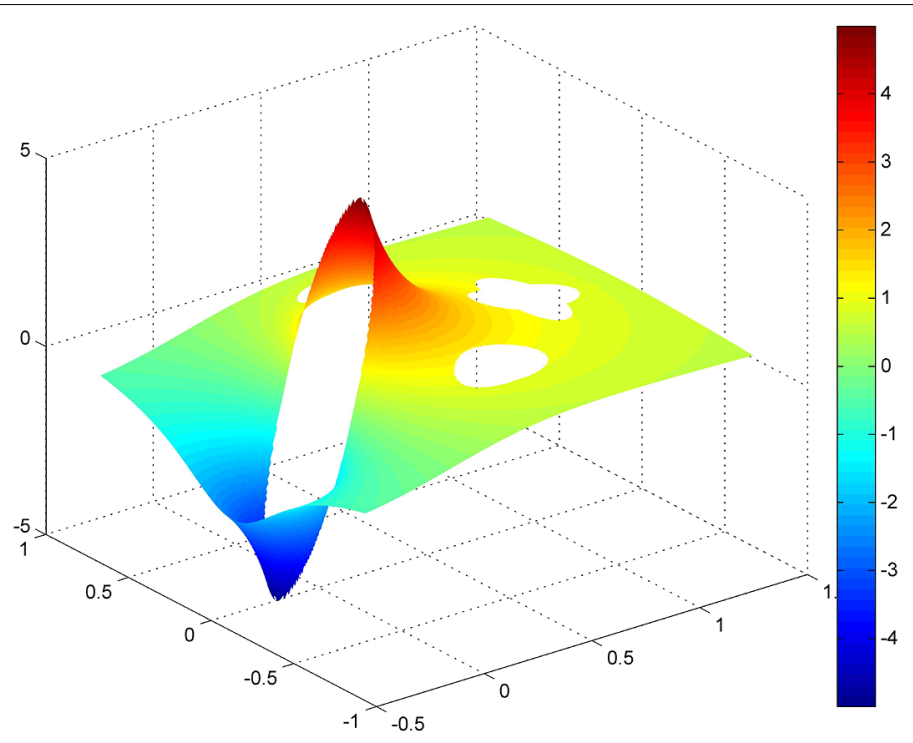

Figure 10 The surface plot of $u_{n}(z)$ for Example 4 with $n=128$.

Table 7 lists the maximum error norms $\left\|u(\eta(t))-u_{n}(\eta(t))\right\|_{\infty}$, where $n$ is the number of nodes and $u_{n}(\eta(t))$ is the numerical approximation of $u(\eta(t))$ based on our method. The errors $\left\|f(z)-f_{n}(z)\right\|$ at some selected points are listed in Table 8 . The absolute errors $\left|u(z)-u_{n}(z)\right|$ for selected points in the entire domain are plotted in Figure 9. Figure 10 shows the surface plot of $u_{n}(z)$ with $n=128$.

\section{Conclusion}

A boundary integral equation for solving both bounded and unbounded Robin problem in multiply connected regions is presented. Unlike previous work on the mixed boundary value problem where the Dirichlet and Neumann conditions are given separately, see [5] 
and [6], the Robin problem has a single boundary condition that is a linear combination of the Dirichlet and Neumann. Here, the method reduces the Robin problem to a RH problem, which leads to a system of integral equations. The proof that these integral equations are linearly independent was shown here. Differential equations were also constructed to provide additional conditions to make the Robin problem uniquely solvable. The integral equations were discretized by the Nyström method with the trapezoidal rule and Wittich's method, while the differential equations were discretized by the five-point central difference method. The presented numerical results illustrate that the proposed method can be used to produce approximations of high accuracy.

\section{Competing interests}

None of the authors have any competing interests in the manuscript.

\section{Authors' contributions}

All authors contributed to the manuscript and typed, read, and approved the final manuscript.

\section{Acknowledgements}

The authors would like to thank the editor and referees for their helpful comments and suggestions which improved the presentation of the paper. The authors also would like to thank the Malaysian Ministry of Education and Research Management Centre (RMC), Universiti Teknologi Malaysia for the partial funding through the fundamental research grant scheme (FRGS) vote R.J130000.7809.4F637.

Received: 20 December 2015 Accepted: 26 April 2016 Published online: 03 May 2016

\section{References}

1. Heiskanen, W, Moritz, H: Physical Geodesy. Freeman, San Francisco (1966)

2. Fang, W, Suxing, Z: Numerical recovery of Robin boundary from boundary measurements for the Laplace equation. J. Comput. Appl. Math. 224, 573-580 (2009). doi:10.1016/j.cam.2008.05.048

3. Gustafson, K, Takehisa, A: The third boundary condition - was it Robin's? Math. Intell. 20(1), 63-71 (1998)

4. Sneddon, IN: Mixed boundary value problems. In: Potential Theory. North-Holland, Amsterdam (1966)

5. Nasser, MMS, Murid, AHM, Ismail, M, Alejaily, EMA: Boundary integral equations with the generalized Neumann kernel for Laplace's equation in multiply connected regions. Appl. Math. Comput. 217, 4710-4727 (2011). doi:10.1016/j.amc.2010.11.027

6. Alhatemi, SAA, Murid, AHM, Nasser, MMS: Solving a mixed boundary value problem via an integral equation with generalized Neumann kernel on an unbounded multiply connected region. Malaysian J. Fund. Appl. Sci. 8(4), 193-197 (2012). doi:10.11113/mjfas.v8n4.147

7. Nasser, MMS: Numerical conformal mapping via a boundary integral equation with the generalized Neumann kernel. SIAM J. Sci. Comput. 31, 1695-1715 (2009). doi:10.1137/070711438

8. Wegmann, R, Murid, AHM, Nasser, M: The Riemann-Hilbert problem and the generalized Neumann kernel. J. Comput. Appl. Math. 182, 388-415 (2005). doi:10.1016/j.cam.2004.12.019

9. Wegmann, R, Nasser, MMS: The Riemann-Hilbert problem and the generalized Neumann kernel on multiply connected regions. J. Comput. Appl. Math. 214, 36-57 (2008). doi:10.1016/j.cam.2007.01.021

10. Petrila, T: Complex value boundary element method for some mixed boundary value problems. Stud. Univ. Babes-Bolyai Inform. 44, 37-42 (1999)

11. Mattheij, RMM, Rienstra, SW, ten Thije Boonkkamp, JHM: Partial Differential Equations: Modeling, Analysis, Computation. SIAM, Philadelphia (2005)

12. Howison, S: Practical Applied Mathematics: Modelling, Analysis, Approximation. Cambridge University Press, Cambridge (2005)

13. Kellogg, OD: Foundations of Potential Theory. Ungar, Cambridge (1929)

14. Salsa, S: Partial Differential Equations in Action: From Modeling to Theory. Springer, Milano (2008)

15. Symm, GT: The Robin problem in a multiply-connected domain. In: Brebbia, CA (ed.) Boundary Element Methods in Engineering. Proceedings of the Fourth International Seminar, pp. 89-100. Springer, Berlin (1982)

16. Nasser, MMS: The Riemann-Hilbert problem and the generalized Neumann kernel on an unbounded multiply connected regions. The University Researcher (IBB University Journal) 20, 47-60 (2009)

17. Gakhov, FD: Boundary Value Problem. Pergamon Press, Oxford (1966)

18. Hamzah, ASA: Solving Robin Problem Via Integral Equations with the Generalized Neumann Kernel. Master's Thesis, Universiti Teknologi Malaysia (2013)

19. Atkinson, KE: The Numerical Solution of Integral Equations of the Second Kind. Cambridge University Press, Cambridge (1997)

20. Gaier, D: Konstruktive Methoden der Konformen Abbildung. Springer, Berlin (1965)

21. Abramowitz, M, Stegun, IE: Handbook of Mathematical Functions, with Formulas, Graphs, and Mathematical Tables. Dover, New York (1965)

22. Helsing, J, Ojala, R: On the evaluation of layer potentials close to their sources. J. Comput. Phys. 227, 2899-2921 (2008)

23. Swarztrauber, PN: On the numerical solution of the Dirichlet problem for a region of general shape. SIAM J. Numer. Anal. 9(2), 300-306 (1972) 